\title{
Review of the Phaonia pallida group (Diptera: Muscidae)
}

\section{Обзор видов группы Pbaonia pallida (Diptera: Muscidae)}

\author{
Nikita E. Vikhrev, Elena A. Erofeeva \\ Никита Е. Вихрев, Елена А. Ерофеева
}

Zoological Museum of Moscow University, Bolshaya Nikitskaya 2, Moscow 125009, Russia. E-mail: nikita6510@ya.ru
Зоологический музей МГУ им. М.В. Ломоносова, Большая Никитская ул., 2, Москва 125009, Россия.

KEY WORDS: Phaonia, Muscidae, Diptera, Palaearctic.

КЛЮЧЕВЫЕ СЛОВА: Phaonia, Muscidae, Diptera, Палеарктика.

ABSTRACT. New and detailed key for the Phaonia pallida group is offered. The variability of species of the $P$. pallida group is considered. Hitherto unknown female of $P$. kowarzii Schnabl, 1887 is described. The distribution of several species of the $P$. pallida group is specified.

РЕЗЮМЕ. Предложен новый и подробный ключ для видов группы Phaonia pallida. Рассмотрена вариабельность видов группы $P$. pallida. Описана неизвестная самка P. kowarzii Schnabl, 1887. Уточнено распространение рассмотренных видов.

\section{Introduction}

Hennig [1963] included in the Phaonia pallida group six Palaearctic species: Phaonia pallida Fabricius, 1787; P. bitincta Rondani, 1866; P. kowarzii Schnabl, 1887; P. regalis Stein, 1900; P. rufiventris Scopoli, 1763 (= populi Meigen, 1826) and P. subventa Harris, 1780 (= variegata Meigen, 1826).

Zinovjev [1981] added to the group P. luteovittata Shinonaga et Kano, 1971. In the present review we include one more species $P$. rubriventris Emden, 1965. The species was described from N Burma and N India from altitudes of $2000 \mathrm{~m}$ or more, it is the border between the Oriental and Palaearctic regions, this species is also recorded for China [Ma et al., 2002]. Thus, totally eight species are considered.

Chinese authors include in the $P$. pallida group six more species from mountainous localities in Chinese Sichuan and Guizhou provinces [Ma et al., 2002], namely: P. brunneiabdomina Xue et Cao, 1989; $P$. fusciaurea Xue et Feng, 1986; P. lucidula Fang et Fan, 1993; P. luteovittatoida Feng et Ma, 2002; P. mengshanensis Feng, 1993 and P. subluteovittata Ma et Deng, 2002. The short key for Chinese species available in English does not clarify enough the situation. Note also that these species are described from very limited series or only from single holotype, so the variability is unknown. Therefore we do not consider those Chinese species in our paper.

The monophyly of the $P$. pallida group seems to us doubtful. Its only apomorphy is a yellow colour of the abdomen, while other characters are very diverse. We prefer to use generally accepted interpretation of the $P$. pallida group till molecular phylogenetic clarifies the relationship between species of Phaonia RobineauDesvoidy, 1830.

The main task of the present review of the P. pallida group is to offer a completely new and detailed key and we hope that our colleagues will found it useful. We also characterize the variability and give direct comparison of two of the most common and at the same time the most variable species $P$. rufiventris and $P$. subventa. A hitherto unknown female of $P$. kowarzii is described. The distribution of several species of the P. pallida group in the eastern part of Palaearctic is specified. Some information on imaginal ecology of species of the group is given.

\section{Material and methods}

The specimens examined in this study are deposited in the following institutions:

BMNH - Natural History Museum, London, UK;

ISEA - Institute of Systematics and Ecology of Animals, Novosibirsk, Russia;

OUMNH - Oxford University Museum of Natural History, UK;

ZIN - Zoological Institute, St-Petersburg, Russia;

ZMUC - Zoological Museum, University of Copenhagen, Denmark;

ZMUM - Zoological Museum of Moscow University, Russia.

Localities are given as follows: country, region, geographical coordinates in the decimal degrees format.

How to cite this article: Vikhrev N.E., Erofeeva E.A. 2018. Review of the Phaonia pallida group (Diptera: Muscidae) // Russian Entomol. J. Vol.27. No.3. P.315-322. doi: 10.15298/rusentj.27.3.14 
The following generally accepted abbreviations for morphological structures are used: $f 1, t 1, f 2, t 2, f 3, t 3=$ fore-, mid-, hind- femur or tibia respectively; $a c$ acrostichal setae; $d c$ - dorsocentral setae; $a, p, d, v=$ anterior, posterior, dorsal, ventral seta(e); prst - presutural, post - postsutural. YPT - yellow pan trap.

\section{Taxonomical account \\ Phaonia bitincta Rondani, 1866 Figs 8-10.}

Phaonia bitincta Rondani, 1866. Type locality: "in the hills of the Parma countryside" (Pont, 1986).

MATERIAL. RUSSIA, Krasnodar reg., S Ozereevka $\left(44.68^{\circ} \mathrm{N}\right.$ $\left.37.63^{\circ} \mathrm{E}\right)$, 14.07.2001, K. Tomkovich, $10^{7}$ (ZMUM); UKRAINE, Kharkov reg., V. Yaroshevsky, 1 우 (ZIN).

DISTRIBUTION. Europe, the easternmost known record is listed above: Krasnodar region, S Ozereevka $\left(37.63^{\circ} \mathrm{E}\right)$.

DISCUSSION. P. bitincta is a rare European species. For example, Hennig [1963] had seen apart from the holotype only 2 specimens (collected by Paul Stein in Germany, Bad Kissingen). The only character used in Hennig's [1963] key to separate $P$. bitincta from $P$. subventa was the length of the aristal hairs. The difference in the scutal pattern was firstly offered by d'Assis-Fonseca [1968]. Gregor et al. [2002] used the number of setae on $t 2$ and yellow pedicel in P. biticta males. We found all these characters more or less useful, add several more and summarized them in the key given below. Considering that our material of $P$. bitincta is very limited, we asked Dr. Verner Michelsen, Denmark and Dr. Adrian Pont, UK to check the key characters against available material of $P$. bitincta in ZMUC and OUMNH respectively (there are 3 specimens in ZMUC collection: $10^{7}$ from France, Alsace prov., $20^{7} \sigma^{7}$ from Spain, Cuenca prov. and $2+9$ from UK in OUMNH collection). Our colleagues kindly confirmed that the key characters works on their specimens too. The presence of the golden setae on the lower occiput in $P$. bitincta was reported by Dr. Michelsen (pers. com.).

Phaonia kowarzii Schnabl, 1887 Figs 11-12.

Phaonia kowarzii. Schnabl, 1887. Type locality: Belarus, Mogilev government (presently Vitebsk reg., Liozno disrt., Zachernya vill. $\left(54.92^{\circ} \mathrm{N} 30.77^{\circ} \mathrm{E}\right)$.

MATERIAL. Syntype, ơ Aricia kowarzii Schnabl Conf. A. C. Pont 2000 (ZIN). RUSSIA: Amur reg.: Zeya env. $\left(53.74^{\circ} \mathrm{N} 127.26^{\circ} \mathrm{E}\right)$ A. Shatalkin, 5.08.1981, $10^{7} ; 19.07 .1982,10^{7} ; 15.08 .1982,1$ ㅇ (all ZMUM); Tukuringra Range, upper reaches of Erakingra River $54.1^{\circ} \mathrm{N} 126.9^{\circ} \mathrm{E}, 19.06 .1957$, A. Zinovjev, 1 ㅇ (ZIN); Khabarovsk reg.: Khabarovsk env., $48.6^{\circ} \mathrm{N} 135.1^{\circ} \mathrm{E}, 2-13.06 .2014$, N. Vikhrev, 2 우 (ZMUM); Bolshekhekhtsirsky NP, $48.267^{\circ} \mathrm{N} 134.767^{\circ} \mathrm{E}, 100$ m, 30.09-1.10.2011, V. Dubatolov, 19 (ISEA); Sopka Dva Brata $\left(48.309^{\circ} \mathrm{N} 135.077^{\circ} \mathrm{E}\right)$, poplar forest, 26.07.1978, D. Kasparyan, $10^{\top}, 1$ ( ZIN); Krasnodar reg.: Agursky Canyon, $43.549^{\circ} \mathrm{N} 39.814^{\circ} \mathrm{E}$, 8-15.05.2018, E. Erofeeva, $20^{7} \sigma^{\top}$; Moscow reg.: Ruza env., $55.66^{\circ} \mathrm{N}$ $36.05^{\circ} \mathrm{E}$, E. Erofeeva, 1-10.06.2016, 1 우 $1-10.06 .2017,4 \sigma^{\top} \sigma^{\top}, 4$ 우;

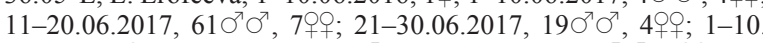

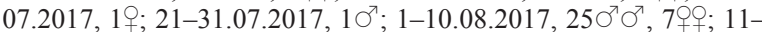
20.08.2017, $16 \sigma^{\top} \sigma^{\top}, 6+0$; $21-31.08 .2017,8 \sigma^{\top} \sigma^{\top}, 3$ 3 + ; $; 1-10.09 .2017$, $2 \sigma^{\top} \sigma^{\top}, 2$, + ; $11-20.09 .2017,1$; ; Dmitrov distr., Kostino env. $\left(56.31^{\circ} \mathrm{N}\right.$ $\left.37.75^{\circ} \mathrm{E}\right)$, N. Vikhrev, 9.06.2009, 1 \% ; 21.07.2009, $10^{7}$; Naro-Fominsk env., $55.393^{\circ} \mathrm{N} 36.784^{\circ} \mathrm{E}, 12.07 .2008$, D. Gavryushin, $10^{7}$ (all ZMUM); Perm reg., Kungur, Uchleskhoz = Preduralie NP (presently abolished), $57.36^{\circ} \mathrm{N} 57.14^{\circ} \mathrm{E}$, A. Zinovjev, 24-30.06.1979, $2 \sigma^{7} \sigma^{7}$ 9.07.1979, $2 \sigma^{7} \sigma^{7}$ (all ZIN); Primorsky reg., Kamenushka env. (43. $\left.622^{\circ} \mathrm{N} 132.232^{\circ} \mathrm{E}\right), 7.06 .1979$, A. Zinovjev, 1 우 12.08 .1984 , A. Shatalkin, $10^{7}$; 14.06.1985, A. Ozerov, 10'; Lazovsky NP., TaChingouz Bay $\left(43.02^{\circ} \mathrm{N} 134.12^{\circ} \mathrm{E}\right), 28.08 .1948$, V. Gussakovsky,
1웅 Khanka Lake, $45.06^{\circ} \mathrm{N} 131.99^{\circ} \mathrm{E}, 15-19.06 .2014$, N. Vikhrev, $10^{\top}$ (all ZMUM); Vladivostok, Sedanka, $43.2^{\circ} \mathrm{N} 132.0^{\circ} \mathrm{E}, 1.06$

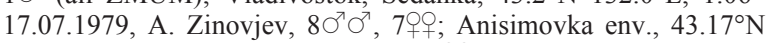
$132.79^{\circ} \mathrm{E}, 17.06 .1979$, A. Zinovjev, 3 우 (all ZIN); Sakhalin reg.: Kunashir Isl., Yuzhno-Kurilsk env. (44.0 N 145.8 $\left.{ }^{\circ} \mathrm{E}\right), 4-7.07 .1979$, A. Zinovjev, $8 \sigma^{7} \sigma^{7}, 2$, 90 ; Sakhalin Isl., $12 \mathrm{~km} \mathrm{~S}$ of Kholmsk, Pravda $\left(46.94^{\circ} \mathrm{N} 142.01^{\circ} \mathrm{E}\right), 25.07 .1971$, E. Narchuk, $20^{7} \sigma^{7}$ (all ZIN); $\mathrm{Za}$ baykalsky reg., Soktuy env. $\left(50.08^{\circ} \mathrm{N} 117.78^{\circ} \mathrm{E}\right), 19.07 .1958, \mathrm{~K}$. Grunin, 1 을 (ZIN).

DISTRIBUTION. From W Europe to Japan, but probably absent in Siberia. It seems that $P$. kowarzii prefers cool oceanic climate like that in Scandinavia or the Pacific Far East. This point of view is indirectly confirmed by our observations in Moscow region where only 3 specimens were collected in period 2008-2016, whereas the year 2017, with abnormally rainy and cold summer, provided a large series of P. kowarzii.

DESCRIPTION OF FEMALE. In contrast to an usual situation, females of $P$. kowarzii are less represented in ZMUM and ZIN collections than males.

A small Phaonia with body length 5.5-6.0 mm. Head. Eyes covered with short and sparse hairs. Frons broad, 1/3 head width, dark; without interfrontal setae. Fronto-orbital plates, parafacials, face and gena dark grey dusted. Frontoorbital plates with 3-4 inclinate and 2 reclinate setae, outer rows with 4-6 short hairs. Pedicel and base of postpedicel dirty yellowish, the rest of postpedicel dark, sometimes antenna entirely dark. Arista yellow in basal $1 / 3$, the rest dark; longest aristal hairs as long as or slightly longer than width of postpedicel. Mouthedge not protruding. Palpi yellow, at apex as wide as width of postpedicel. Thorax. Disc of scutum mostly black with a pair of submedian grey vittae inside of $d c$ rows; sides of scutum outside the intraalar rows typically yellow to translucent yellow. Scutellum usually entirely yellow, sometimes dark at base. Upper pleura (anepisternum and anepimeron) mostly yellow, lower pleura (katepisternum and meron) mostly dark (Fig. 12). Dark specimens with only postpronotal lobe yellow (as in male) are also not rare (about 30\% females). Thoracic spiracles yellow, posterior one small, shorter than width of katepimeron. Prosternum, notopleuron, katepimeron and meron bare; $d c 2+3$, all strong; ac 0+1; prealar seta shorter than posterior notopleural. Wings with a slight yellow tint; veins bare; crossveins not infuscated; calypters and halter yellow. Legs, including coxae, yellow, only tarsi dark. $t 1$ without seta. $t 2$ with $3 p$ setae. $f 3$ with 3-4 av near apex. $t 3$ with 3-4 av, 2 ad and $1 p d$ setae. Abdomen yellow with thin whitish dusting and indistinct narrow median vitta; apex of abdomen may be more or less darkened. Sternite 1 bare. Tergite 4 and 5 with distinct discal and marginal setae.

Male (Fig. 11) differs from female as follows: Frontoorbital plates touching except for in anterior and posterior quarters; 3-4 inclinate setulae restricted to anterior quarter of frons, reclinate setae absent. Anterior facets of eyes enlarged. Postpedicel remarkably yellowish-white. Palpi narrower, half as wide as postpedicel. Scutum and pleura dark, only postpronotal lobe translucent yellow; scutellum darkened basally.

\section{Phaonia luteovittata Shinonaga et Kano, 1971}

Figs 2-3.

Phaonia luteovittata Shinonaga et Kano, 1971. Type locality: Japan, Hokkaido.

MATERIAL. RUSSIA: Chelyabinsk reg., Taganay Mts (55.221 $\left.{ }^{\circ} \mathrm{N} 59.734^{\circ} \mathrm{E}\right), 18-24.07 .2008, \mathrm{~K}$. Tomkovich, $10^{7}$ (ZMUM); Kemerovo reg., Teba $\left(53.64^{\circ} \mathrm{N} 88.57^{\circ} \mathrm{E}\right), 9.07 .1962, \mathrm{~K}$. Grunin, 1 우 $(\mathrm{ZIN})$; Perm reg., Kungur, Uchleskhoz $=$ Preduralie NP (presently 
abolished, $\left.57.36^{\circ} \mathrm{N} 57.14^{\circ} \mathrm{E}\right), 16.07 .1979$, A. Zinovjev, $10^{7}(\mathrm{ZIN})$; Primorsky reg.: $20 \mathrm{~km} \mathrm{~S}$ of Oblachnaya $\mathrm{Mt}\left(43.69^{\circ} \mathrm{N}, 134.21^{\circ} \mathrm{E}\right)$ 8.08.1963, E. Narchuk, 1 을 (ZIN); SW bank of Khanka L. $\left(45.0^{\circ} \mathrm{N}\right.$ $\left.132.0^{\circ} \mathrm{E}\right), 26.07 .1969$, M. Kandybina, $10^{7}$ (ZIN); Sakhalin reg.: Kunashir Isl., Tretyakovo env., $43.99^{\circ} \mathrm{N} 145.66^{\circ} \mathrm{E}$, YPT, $13-$ 22.09.2009, I. Melnik, $10^{7}$, 5우 Kunashir Isl., Peschanoe Lake, $43.950^{\circ} \mathrm{N} 145.593^{\circ} \mathrm{E}, 1-5.08 .2008$, I. Melnik, $10^{7}$; Kordon Filatovsky env. $\left(44.194^{\circ} \mathrm{N} 146.027^{\circ} \mathrm{E}\right)$, YPT, 2-3.09.2009, I. Melnik, 2 우 (all ZMUM); Kunashir Isl., Lagunnoe L. (44.05 ${ }^{\circ} \mathrm{N} 145.77^{\circ} \mathrm{E}$ ), Violovich, 17-23.08.1955, 10', 1 ㅇ (ZIN); Sakhalin Isl., Novoaleksandrovsk $\left(47.05^{\circ} \mathrm{N} 142.72^{\circ} \mathrm{E}\right), 22.07 .1968$, E. Narchuk, $1 \sigma^{\top}(\mathrm{ZIN})$ Shikotan Isl., Tserkovny Bay, $43.75^{\circ} \mathrm{N} 146.70^{\circ} \mathrm{E}$, Yu. Sundukov,

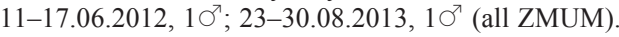

DISTRIBUTION. Known from Japan (Hokkaido and Honsu) and Russia: from the Far East (Primorsky and Sakhalin reg.) to Ural (Chelyabinsk and Perm reg.).

DISCUSSION. The original description of $P$. luteovittata was reproduced in a more accessible source [Shinonaga, 2003: 163]. We have 2 specifications to the description: katepimeron bare [Shinonaga, 2003] or with setula (in about $40 \%$ of males and 15\% females) (1); all females have no prst ac (2). Thus, $P$. luteovittata is very similar to European $P$. rufiventris, these species differ by body coloration (Fig. 2) and shape of male cerci (Fig. 3). Typically P. luteovittata has: $f 1$ darkened brown; tergites 4 and 5 entirely glossy black, tergite 3 often darkened on posterior border; disc of scutum subglossy, with only very thin grey dusting; colour of thorax variable: from almost entirely black to presence of some yellowish-brown areas on postpronotal lobe, postsutural sides of scutum and pleura; scutellum is always pure amber-yellow.

In specimens collected west of the Far East the colour pattern is slightly less distinct, but still reliable. For example, based on the colour pattern one of two males collected in Chelyabinsk region was identified as $P$. rufiventris, while another one as $P$. luteovittata, subsequent examination of genitalia confirmed correctness of both identifications. It seems that both European $P$. rufiventris and Far Eastern $P$. luteovittata are present in Siberia and Ural, but both species are uncommon in sharply continental climate of central Palaearctic. Further collecting can clarify the problem.

Phaonia pallida Fabricius, 1787 Figs 13-15.

Phaonia pallida Fabricius, 1787. Type locality: Copenhagen, Denmark.

MATERIAL, ABKHAZIA, Sukhumi $\left(43^{\circ} \mathrm{N} 41^{\circ} \mathrm{E}\right)$ env. 9.06.1932, B. Rodendorf, $10^{7}$ (ZMUM); ARMENIA, Ijevan env. Verin-Ahgdan $=$ Gandzakar $\left(40.84^{\circ} \mathrm{N} 45.17^{\circ} \mathrm{E}\right), 23.07 .1969$, V. Rikhter, $4 \sigma^{7} \sigma^{7}$, 3 우 (ZIN); AZERBAIJAN: Lankoran, Xanbulan Reservoir env. $\left(38.65^{\circ} \mathrm{N} 48.78^{\circ} \mathrm{E}\right), 19-27.10 .2008, \mathrm{~N}$. Vikhrev, 2+o; YPT, 22-28.05.2009, K. Tomkovich, $10^{7}, 2$, 90 (all ZMUM); BELARUS, Brest reg., Belovezhskaya Pushcha NR $\left(52.5^{\circ} \mathrm{N} 23.8^{\circ} \mathrm{E}\right)$, 17.07-7.08.1961, E. Narchuk, 7우 (ZIN); FRANCE, Seine-Maritime, Foret de Brotonne $\left(49.45^{\circ} \mathrm{N}\right.$ 0.75 $\left.{ }^{\circ} \mathrm{E}\right), 12.08 .2009$, G. Pennards, $1+$ (ZMUM); HUNGARY, Balaton L., Tihany Peninsula $\left(46.9^{\circ} \mathrm{N} 17.9^{\circ} \mathrm{E}\right)$, broadleaf forest, 30.06.1970, K. Gorodkov, 3 + (ZIN); GERMANY, Saarbrucken env. $\left(49.2^{\circ} \mathrm{N} 7.0^{\circ} \mathrm{E}\right), 9-13.07$ 1995, K. Gorodkov, $70^{7} \sigma^{7}$ (ZIN); RUSSIA: Crimea reg.: Karadag $\left(44.94^{\circ} \mathrm{N} 35.23^{\circ} \mathrm{E}\right), 22.06 .1982$, L. Zimina, 1 웅 Pionerskoe env $\left(44.88^{\circ} \mathrm{N} 34.20^{\circ} \mathrm{E}\right), 14-17.06 .1975$, A. Zhelokhovtsev, $4 \sigma^{\top} \sigma^{7}, \mathrm{~L}$.

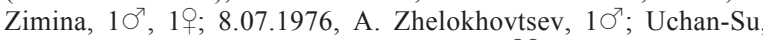
$44.99^{\circ} \mathrm{N} 34.10^{\circ} \mathrm{E}, 30.09 .1915$, N. Vikhrev, 3 우 (all ZMUM); Krasnodar reg.: Adygea, Dakhovskaya env., $44.199^{\circ} \mathrm{N} 40.170^{\circ} \mathrm{E}, 465$

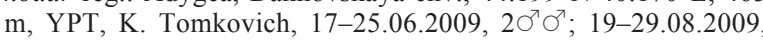
5 우 $44.217^{\circ} \mathrm{N} 40.105^{\circ} \mathrm{E}, 900 \mathrm{~m}, 24-25.06 .2009$, K. Tomkovich, $1 \mathrm{O}^{7}$; Agursky Canyon, $43.549^{\circ} \mathrm{N} 39.814^{\circ} \mathrm{E}, \mathrm{N}$. Vikhrev, 26.10.2006, 2कo ; 29.09.2008, 1 ; ; 27.10.2007, D. Gavryushin, 1; 3-8.06.2008, K. Tomkovich, $30^{7} \sigma^{7}$; Lazarevskoe env. $\left(43.90^{\circ} \mathrm{N} 39.35^{\circ} \mathrm{E}\right), 12.07$. 1989, A. Shatalkin, 1 ; ; S Ozereevka $\left(44.680^{\circ} \mathrm{N} 37.631^{\circ} \mathrm{E}\right)$,
16.07.2001, K. Tomkovich, $10^{7}$ (all ZMUM); Kursk reg., Streletskaya steppe, oak forest $\left(51.565^{\circ} \mathrm{N} 36.085^{\circ} \mathrm{E}\right), 12.08 .2008, \mathrm{~N}$. Vikhrev, 1 (ZMUM); Moscow reg.: Ruza env., $55.66^{\circ} \mathrm{N} 36.05^{\circ} \mathrm{E}$, E. Erofeeva, 11-20.06.2016, 1 $\sigma^{7} ; 21-30.06 .2016,3 \sigma^{7} \sigma^{7} ; 1-10.07$. 2016, 2우; 11-20.07.2016, $2 \sigma^{7} \sigma^{7}$; 11-20.08.2016, 2우; 21$30.06 .2017,1 \sigma^{7} ; 1-10.07 .2017,6 \sigma^{7} \sigma^{7} ; 21-31.07 .2017,4 \sigma^{7} \sigma^{7} ; 1-$

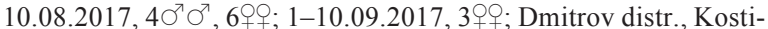
no env. $\left(56.31^{\circ} \mathrm{N} 37.75^{\circ} \mathrm{E}\right), \mathrm{N}$. Vikhrev, 27.06.2008, $10^{7} ; 29.06$. 2012, $1 \mathrm{O}^{7}$; Andreevskoye env., $55.976^{\circ} \mathrm{N} 35.5857^{\circ} \mathrm{E}, 10.07 .2007$, A. Ozerov, $10^{7}, 2+0$ (all ZMUM); Nizhny Novgorod reg., Nizhny Novgorod env., $56.28^{\circ} \mathrm{N} 43.70^{\circ} \mathrm{E}, 12.07 .2009$, N. Vikhrev, 1 우 (ZMUM); Perm reg., Kungur, Uchleskhoz $=$ Preduralie NP (presently abolished), $57.36^{\circ} \mathrm{N} 57.14^{\circ} \mathrm{E}$, Borisova, 8-15.07.1957, $4 \sigma^{\top} \sigma^{\top}$,

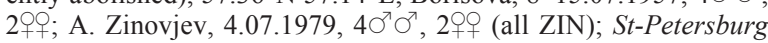
reg., Kiperort peninsula, $60.48^{\circ} \mathrm{N} 28.56^{\circ} \mathrm{E}, 15-25.05 .2012$, K. Tomkovich, $7 \sigma^{\top} \sigma^{\top}, 1+$ (ZMUM); Saratov reg., Saratov env. $\left(51.6^{\circ} \mathrm{N}\right.$ $\left.46.1^{\circ} \mathrm{E}\right), 1-21.07 .1917$, A. Stackelberg, 2 +o (ZIN); Voronezh reg., Borisoglebsk env., $51.397^{\circ} \mathrm{N} 42.014^{\circ} \mathrm{E}, 23.08 .2012$, K. Tomkovich, 1 ; ; Voronezhsky NP $\left(51.9^{\circ} \mathrm{N} 39.6^{\circ} \mathrm{E}\right), 20.06 .1957$, S. Kelejnikova, $10^{7}$ (all ZMUM); SERBIA: Crni Vrh env., $43.407^{\circ} \mathrm{N} 22.587^{\circ} \mathrm{E}, 800$ m, N. Vikhrev, 16-22.09.2014, 1; 1-7.07.2015, 3 $0^{\top} \sigma^{\top}, 1$ 우 29.06.2015, A. Ozerov et M. Krivosheina, $3 \sigma^{7} \sigma^{7}, 19$ (all ZMUM); TURKEY: Duzce prov., Aktas Selale (41.01 $\left.{ }^{\circ} \mathrm{N} 31.04^{\circ} \mathrm{E}\right), 400 \mathrm{~m}, 20$ 21.06.2010, N. Vikhrev, $10^{7}$; Sakarya prov., Karasu $\left(41.03^{\circ} \mathrm{N} 30\right.$. $\left.79^{\circ} \mathrm{E}\right), 15.06 .2010$, N. Vikhrev , 1 \%; Zonguldak prov., Alapl env. $\left(41.14^{\circ} \mathrm{N} 31.36^{\circ} \mathrm{E}\right), 28.08 .2009$, N. Vikhrev, 19 (all ZMUM); UKRAINE: Zakarpatsky reg., Turya Polyana $\left(48.7^{\circ} \mathrm{N} 22.804^{\circ} \mathrm{E}\right)$, 25-29.06.1964, L. Zimina, $3 \sigma^{7} \sigma^{\top}, 19$ (ZMUM); Poltava reg., Yareski (49.84 $\left.{ }^{\circ} \mathrm{N} 33.90^{\circ} \mathrm{E}\right), 06-07.1929$, Gildebrandt, $20^{\top} \sigma^{\top}, 30$, 90 (ZIN).

DISTRIBUTION. All over Europe; Iran and Israel [Pont, 1986]. The only Siberian record (Tyumen reg., Russia [Veselkin, 1966]) seems to us doubtful and we had not found any specimens of $P$. pallida among bequest to ZMUM Veselkin's collection. During several years of intensive collecting in Altai Mts and Novosibirsk region $P$. pallida was never recorded. Thus the easternmost reliable record is W Ural, Perm region, $57^{\circ} \mathrm{E}$.

REMARKS. Normally $P$. pallida has entirely yellow pleura and scutum (the latter with some whitish dusting) as on Figs 14-15, but rarely pleura and scutum may be extensively darkened as on Fig. 13.

\section{Phaonia regalis Stein, 1900}

Phaonia regalis Stein, 1900. Type locality: Tiflis = Tbilisi, Georgia.

MATERIAL. GEORGIA: Tiflis = Tbilisi distr., Kojori, $41.66^{\circ} \mathrm{N}$ 44.71 ${ }^{\circ} \mathrm{E}, 20.06 .1916$, Andrievsky, $10^{7}$ (ZIN); Abastumani, pine forest with oak, $41.75^{\circ} \mathrm{N} 42.88^{\circ} \mathrm{E}, 10.09 .1982$, D. Kasparyan, 1 우 (ZIN); Manglisi $\left(41.69^{\circ} \mathrm{N} 44.42^{\circ} \mathrm{E}\right), 1-10.08 .1900, \mathrm{~S}$. Zacharov, 1 ㅇ (ZMUM); GREECE, C. Macedonia, Kerkini L. env., Wine Trap, $41.285^{\circ} \mathrm{N} 23.208^{\circ} \mathrm{E}, 475 \mathrm{~m}, 9-15.06 .2008$, G. Ramel, $10^{\top}, 1$ 우 (ZMUM).

DISTRIBUTION. An uncommon species known from few localities in SW Palaearctic: Austria, Bulgaria, Greece, Georgia and Turkey.

REMARKS. Female from Greece has 1 pair of rather weak but distinct prst ac.

\section{Phaonia rubriventris Emden, 1965} Figs 16-17.

Phaonia rubriventris Emden, 1965. Type locality: N Burma (Myanmar), Kambaiti and N India, Darjeeling.

MATERIAL. VIETNAM, Lai Chau prov., Hoang Lien NP, $22.350^{\circ} \mathrm{N} 103.768^{\circ} \mathrm{E}, 1950 \mathrm{~m}, 11.04 .2012$, D. Gavryushin, $1 \sigma^{7}$ (ZMUM).

DISTRIBUTION. Known from N India, N Burma, N Vietnam (new record) and China [Ma et al., 2002].

REMARKS. We could add to detailed Emden's [1965] description only that in $P$. rubriventris both meron and katepimeron are bare; prealar seta is strong; radial node of 
wing is bare (thou, that follows from the key for Oriental Phaonia [Emden, 1965: 218]).

\section{Phaonia rufiventris Scopoli, 1763} Fig. 4.

P. rufiventris Scopoli, 1763. Type locality: Carniolia (presently Slovenia).

Phaonia populi Meigen, 1826.

MATERIAL. AZERBAIJAN, Lankoran, Xanbulan Reservoir env., $38.65^{\circ} \mathrm{N} 48.78^{\circ} \mathrm{E}, \mathrm{YPT}, 18-20.05 .2009, \mathrm{~K}$. Tomkovich, $10^{\top}$ (ZMUM) BELARUS, Brest reg., Belovezhskaya Pushcha NR $\left(52.5^{\circ} \mathrm{N} 23.8^{\circ} \mathrm{E}\right)$, 7.08.1961, E. Narchuk, $130^{7} 0^{7}, 23$ 우 (ZIN); FINLAND, Finland env., Harjavalta $\left(61.3^{\circ} \mathrm{N} 22.1^{\circ} \mathrm{E}\right), 25.06 .2009$, T. van Ooik, 1 ( $(\mathrm{ZMUM})$ RUSSIA: Altai Republic: Shebalinsky distr., Ilyinka env., $51.45^{\circ} \mathrm{N}$ $85.14^{\circ} \mathrm{E}, 1100 \mathrm{~m}, 19.07 .2011$, V. Sorokina, 10 $0^{7}$; Turochak distr, Obogo env. (51.52 $\left.{ }^{\circ} \mathrm{N} 87.29^{\circ} \mathrm{E}\right), 900 \mathrm{~m}, 28.06 .2003$, V. Sorokina, $10^{\circ}$ (all ISEA); Chelyabinsk reg., Taganay Mts $\left(55.221^{\circ} \mathrm{N} 59.734^{\circ} \mathrm{E}\right), 18$ 24.07.2008, K. Tomkovich, $10^{7}$ (ZMUM); Crimea reg.: AlushtaRybachye, $44.7-8^{\circ} \mathrm{N} 34.4-6^{\circ} \mathrm{E}, 18-25.04 .2014, \mathrm{~N}$. Vikhrev, $20^{7} \mathrm{O}^{7}$ Bolshoy Canyon, $44.519^{\circ} \mathrm{N} 33.996^{\circ} \mathrm{E}, 540 \mathrm{~m}, 26-30.09 .2015$, N Vikhrev, $20^{\top} O^{7}, 2$ OPO ; Yalta env., $44.44^{\circ} \mathrm{N} 34.12^{\circ} \mathrm{E}, 300 \mathrm{~m}, 4-9.05 .2016$, E. Erofeeva, 10 ; N. Vikhrev, $10^{7}$ (all ZMUM); Kemerovo reg., W Kuznetsky Alatau, Rybnoye Lake, $\left(54.33^{\circ} \mathrm{N} 88.40^{\circ} \mathrm{E}\right), 1240 \mathrm{~m}, 9.08$ 2006, D. Lopatin, $10^{7}$ (ISEA); Krasnodar reg.: Agursky Canyon, $43.548^{\circ} \mathrm{N} 39.815^{\circ} \mathrm{E}, 20-24.04 .2008$, A. Ozerov, 2우, D. Gavryushin,

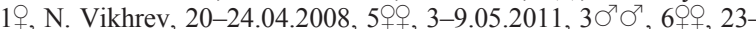
25.06.2011, $10^{\top}$; Guzeripl, $44.004^{\circ} \mathrm{N} 40.134^{\circ} \mathrm{E}, 700 \mathrm{~m}, 5-7.05 .2013$, N. Vikhrev, $10^{\prime}, 2$, 9 ; Lagonaki Plateau, $44.012^{\circ} \mathrm{N} 40.034^{\circ} \mathrm{E}, 1020$ $1500 \mathrm{~m}$, N. Vikhrev, 27-30.06.2011, 10', 1; Lagonaki Plateau, Azish cave env., $44.107^{\circ} \mathrm{N} 40.020^{\circ} \mathrm{E}, 1450 \mathrm{~m}$, YPT, 26-28.09.2009, K. Tomkovich, 2 우; Dakhovskaya env., $44.220^{\circ} \mathrm{N} 40.100^{\circ} \mathrm{E}, 750-950$ m, YPT, 24.06.2009, K. Tomkovich, 107, 2 99 (all ZMUM); Krasnoyarsk reg., Krasnoyarsk env., E bank, Stolby, $55.963^{\circ} \mathrm{N} 92.745^{\circ} \mathrm{E}$ 209-260 m, YPT, 18-19.06.2011, K. Tomkovich, 19 (ZMUM); Kursk reg.: Centralno-Chernozemny NP., Streletskaya steppe $\left(51.565^{\circ} \mathrm{N}\right.$ $\left.36.085^{\circ} \mathrm{E}\right), 7.09 .2007$, A. Ozerov, $10^{7}$ (ZMUM); Moscow reg.: Ruza env., $55.66^{\circ} \mathrm{N} 36.05^{\circ} \mathrm{E}$, E. Erofeeva, $11-20.09 .2015,3$ 웅 $1-10.06$

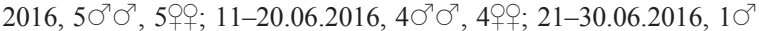

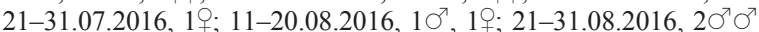

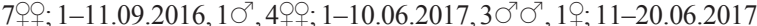
$150^{7} 0^{7}$; 21-30.06.2017, $90^{7} \sigma^{7}, 3$ 우우; 1-10.07.2017, $20^{7} \sigma^{7}, 5$ 우우 $21-$ 31.07.2017, $20^{7} \sigma^{\top} ; 1-10.08 .2017,50^{7} \sigma^{7}, 26$ 우우; $11-20.08 .2017,10$ 우우

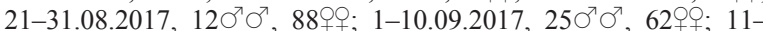
20.09.2017, $20^{7} 0^{7}$, 2qoq; Dmitrov distr., Kostino env. $\left(56.31^{\circ} \mathrm{N}\right.$ $\left.37.75^{\circ} \mathrm{E}\right), \mathrm{N}$. Vikhrev, $15.06 .2006,1$ \% $2.06 .2010,10^{7} ; 27.08 .2012$, 19; Moskovsky env., $55.588^{\circ} \mathrm{N} 37.333^{\circ} \mathrm{E}, \mathrm{YPT}, \mathrm{K}$. Tomkovich, 29 31.07.14, $10^{\top} ; 1.07 .2015,1$; $; 23.06 .2016,10^{7}$; Chekhov distr., st. 66 $\mathrm{km}\left(55.229^{\circ} \mathrm{N} 37.487^{\circ} \mathrm{E}\right), 22.05 .2012, \mathrm{~K}$. Tomkovich, $10^{7}$; Podolsk env., $55.448^{\circ} \mathrm{N} 37.563^{\circ} \mathrm{E}, \mathrm{YPT}, \mathrm{K}$. Tomkovich, $25-29.08 .2011,10^{7}$ $2-5.09 .2011,1$ 웅 Abramtsevo $\left(56.184^{\circ} \mathrm{N} 37.968^{\circ} \mathrm{E}\right)$, E. Smirnov, 3.06.1957, $10^{\top}, 25.06 .1958,1$; ; Golitsyno $\left(55.613^{\circ} \mathrm{N} 36.978^{\circ} \mathrm{E}\right)$, 6.06.1981, A. Shatalkin, $10^{7}$ (all ZMUM); Murmansk reg.: Monchegorsk env. $\left(67.946^{\circ} \mathrm{N} 32.895^{\circ} \mathrm{E}\right), \mathrm{M}$. Kozlov, 11-18.07.2009, $10^{\mathrm{\prime}} \cdot 25$ 31.07.2009, 1 ; ; Murmansk env., $68.95^{\circ} \mathrm{N} 33.13^{\circ} \mathrm{E}, 9-15.08 .2010, \mathrm{~N}$. Vikhrev, 2o+p; Murmansk env., $68.973^{\circ} \mathrm{N} 33.138^{\circ} \mathrm{E}, 21.07 .2011$, A Ozerov, $10^{7}$ (all ZMUM); Novosibirsk reg.: Novosibirsk Academy town $\left(54.817^{\circ} \mathrm{N} 83.15^{\circ} \mathrm{E}\right), 14.06 .2008$, O. Kosterin, 1 ; ; Novosibirsk env., $54.87^{\circ} \mathrm{N} 83.05^{\circ} \mathrm{E}, 3.09 .2016$, O. Kosterin, 19 (all ZMUM); St Petersburg reg., Kiperort peninsula, $60.48^{\circ} \mathrm{N} 28.56^{\circ} \mathrm{E}, 15-25.05 .2012$, K. Tomkovich, $30^{7} 0^{7}, 1$ ( (all ZMUM); Tver reg., Derbovezh L. env. $56.33^{\circ} \mathrm{N} 31.97^{\circ} \mathrm{E}, 21.06 .2012$, N. Vikhrev, 19 (ZMUM); Vladimir reg., Vladimir env., $56.05^{\circ} \mathrm{N} 40.41^{\circ} \mathrm{E}, 16.06 .2013$, N. Vikhrev, $4 \bigcirc^{7} \mathrm{O}^{\prime}$ (ZMUM); SERBIA: Crni Vrh env., $43.407^{\circ} \mathrm{N} 22.587^{\circ} \mathrm{E}, 800 \mathrm{~m}, 16$ 22.09 .14 , N. Vikhrev, $80^{7} 0^{7}$, 4 옹 Babin Zub $\left(43.374^{\circ} \mathrm{N} 22.621^{\circ} \mathrm{E}\right)$ 1547 m, 1-7.07.2015, N. Vikhrev, 107, 2웅 A. Ozerov et M. Krivosheina, $30^{7} \sigma^{7}$ (all ZMUM). SWITZERLAND, Graubunden (Grisons) kanton, J. Warner, 28.08.1910, 1; 12.08.1913, $10^{\top}$ (ZIN).

DISTRIBUTION. P. rufiventris is a common species all over Europe. It is also recorded from Ural region; West and Central Siberia, though $P$. rufiventris becomes uncommon in Asia and in spite of intensive collecting it is represented by few Asian specimens in ZMUM, ZIN and ISEA collections.
REMARKS. The variability in comparison with $P$. subventa is given below.

\section{Phaonia subventa Harris, 1780}

Figs 5-7.

Phaonia variegata Meigen, 1826

Phaonia subventa Harris, 1780. Type locality: S E England

MATERIAL. AZERBAIJAN: Lankoran, Xanbulan Reservoir env, $38.65^{\circ} \mathrm{N} 48.78^{\circ} \mathrm{E}, 19-27.10 .2008$, N. Vikhrev, $3 \sigma^{7} \sigma^{7}, 1$ 우 YPT, 15-22.05.2009, K. Tomkovich, 10', 4우 (all ZMUM); BELARUS, Vitebsk reg., Orsha env., Dnepr R., $54.543^{\circ} \mathrm{N} 30.463^{\circ} \mathrm{E}, 11.06 .2017$, N. Vikhrev, $10^{7}, 4$ 우 (ZMUM); GREECE, Kerkini Mts, $41.34^{\circ} \mathrm{N}$ $23.33^{\circ} \mathrm{E}, 27.03 .2008$, G. Ramel, $10^{7}$ (ZMUM); ITALY, 07.1933, Zhenzhurist (N. Filippov), $10^{7}$ (ZMUM); MOROCCO, Al Haouz prov., Oukaimeden $\left(31.23^{\circ} \mathrm{N} 7.81 \mathrm{~W}\right), 2000 \mathrm{~m}, 13-17.05 .2012, \mathrm{~N}$. Vikhrev, $1 \sigma^{7}$ (ZMUM); PORTUGAL, Vila do Conde, $41.35^{\circ} \mathrm{N}$ $8.75^{\circ} \mathrm{W}, 2-6.07 .2010$, O. Kosterin, $3 \circ 0$ (ZMUM); RUSSIA: Crimea reg.: Alupka env. $\left(44.421^{\circ} \mathrm{N} 34.038^{\circ} \mathrm{E}\right), 4.10 .1936$, N. Filippov, 3 우; Alushta-Rybachye, $44.7-8^{\circ} \mathrm{N} 34.4-6^{\circ} \mathrm{E}, 18-25.04 .2014, \mathrm{~N}$. Vikhrev, $4 \circ^{\top} \sigma^{\top}$; Bolshoy Canyon, $44.519^{\circ} \mathrm{N} 33.996^{\circ} \mathrm{E}, 540 \mathrm{~m}$, 26.09.2015, N. Vikhrev, $6 \mathrm{O}^{7} \mathrm{\sigma}^{7}$; Yalta env., $44.44^{\circ} \mathrm{N} 34.12^{\circ} \mathrm{E}, 300 \mathrm{~m}$,

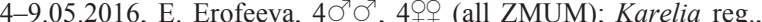
Pudozhsky distr., Tyagozero Lake $\left(62.03^{\circ} \mathrm{N} 36.12^{\circ} \mathrm{E}\right), 6.08 .1969, \mathrm{~V}$. Korolev, 19 (ZMUM); Krasnodar reg.: Adler env., $43.477^{\circ} \mathrm{N}$ $39.907^{\circ} \mathrm{E}, 27.10 .2009$, D. Gavryushin, $20^{7} \sigma^{7}$; Agursky Canyon $\left(43.548^{\circ} \mathrm{N} 39.815^{\circ} \mathrm{E}\right)$, A. Ozerov, 27-30.03.2006, $6 \mathrm{O}^{\top} \mathrm{\sigma}^{\top}, 1+$,

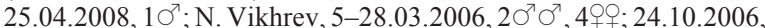

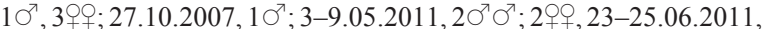
1 웅 Psekhako Mt, $43.697^{\circ} \mathrm{N} 40.367^{\circ} \mathrm{E}, 2000 \mathrm{~m}, 14-18.06 .2008, \mathrm{~K}$. Tomkovich, 2o; Alpika-service, $43.67^{\circ} \mathrm{N} 40.28^{\circ} \mathrm{E}, 600 \mathrm{~m}, 11-$ 13.06.2008, K. Tomkovich, 3ㅇ; Adygea: N Lagonaki Mt, Azish cave env., $44.107^{\circ} \mathrm{N} 40.020^{\circ} \mathrm{E}, 1450 \mathrm{~m}$, YPT, $15-17.06 .2009, \mathrm{~K}$. Tomkovich, 3우; Lagonaki, $44.009^{\circ} \mathrm{N} 39.994^{\circ} \mathrm{E}, 1500-1900 \mathrm{~m}, \mathrm{~N}$. Vikhrev, 27-30.06.2011, 1; 11.06.2012, 1ㅇ; 5-9.06.2015, 1우 Dakhovskaya env., $44.199^{\circ} \mathrm{N} 40.170^{\circ} \mathrm{E}$, YPT, $18-31.08 .2009$, K. Tomkovich, 1 ; ; Kursk reg.: Selihovi Dvori env., Streletskaya steppe, oak forest $\left(51.565^{\circ} \mathrm{N} 36.085^{\circ} \mathrm{E}\right), \mathrm{N}$. Vikhrev, 26.05.2007, $10^{7} ; 12.08 .2008$, 1웅 Centr.-Chernozem Res., Bukreevy Barmy $\left(51.50^{\circ} \mathrm{N} 37.34^{\circ} \mathrm{E}\right)$, 6.05.2008, K. Tomkovich, $10^{7}$ (all ZMUM); Moscow reg., Ruza env, $55.66^{\circ} \mathrm{N} 36.05^{\circ} \mathrm{E}$, E. Erofeeva, 1-11.09.2016, 390; 21-30.06.

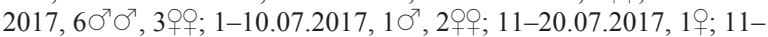
20.08.2017, 6우; 21-31.08.2017, 21 $\sigma^{\top} \sigma^{\top}, 29+9$; 1-10.09.2017, $100^{7} \sigma^{7}, 27$ 우; 11-20.09.2017, 1 우 (all ZMUM); Murmansk reg., Monchegorsk env. $\left(67.946^{\circ} \mathrm{N} 32.895^{\circ} \mathrm{E}\right), 25-31.07 .2009$, M. Kozlov, $10^{\top}$ (ZMUM); Novgorod reg., Holm env. $\left(57.147^{\circ} \mathrm{N} 31.175^{\circ} \mathrm{E}\right)$, 23-30.09.2000, K. Tomkovich, 1 ( (ZMUM); Ryazan reg., Kasimov env., Zalesnoe vill., $54.969^{\circ} \mathrm{N} 41.327^{\circ} \mathrm{E}, 21-26.07 .2013$, N. Vikhrev, 1 ( (ZMUM); SERBIA: Crni Vrh env., $43.407^{\circ} \mathrm{N} 22.587^{\circ} \mathrm{E}, 800 \mathrm{~m}$,

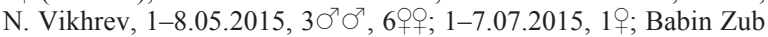
$\left(43.374^{\circ} \mathrm{N} 22.621^{\circ} \mathrm{E}\right), 1547 \mathrm{~m}, 6.07 .2015$, A. Ozerov et M. Krivosheina, $1 \sigma^{7}$ (all ZMUM); TURKEY: Antalya prov.: Kurshunlu waterfall $\left(37.07^{\circ} \mathrm{N} 30.86^{\circ} \mathrm{E}\right), \mathrm{N}$. Vikhrev, 25.02.2008, 2 \%ᄋ; 1.04 .2008 , $10^{7}$; Manavgat, pine forest $\left(36.87^{\circ} \mathrm{N} 31.47^{\circ} \mathrm{E}\right), 19-24.02 .2008, \mathrm{~N}$. Vikhrev, 2o+; 31.03.2008, N. Vikhrev, 10'; K. Tomkovich, 26.02. 2008, $10^{\top}, 1$; ; Avd $n$ prov., Dilek Milli Park $\left(37.7^{\circ} \mathrm{N} 27.2^{\circ} \mathrm{E}\right), 19.12$. 2006 , N. Vikhrev, $10^{7}$; Mersin prov., near Silifke, $36.405^{\circ} \mathrm{N} 33.804^{\circ} \mathrm{E}$, 23.04.2010, N. Vikhrev, 1ㅇ; Sakarya prov., Karasu env., $41.1^{\circ} \mathrm{N}$ $30.7^{\circ} \mathrm{E}, 09.05 .2009$, N. Vikhrev, $10^{7}$ (all ZMUM); UKRAINE, Kanev $\left(49.759^{\circ} \mathrm{N} 31.469^{\circ} \mathrm{E}\right), 19.09 .1975$, V. Sychevskaya, 1 우 (ZMUM); USA, $R I$, Coventry Co, $41.69^{\circ} \mathrm{N} 71.55^{\circ} \mathrm{W}, 1-14.05 .2017$, A. Medvedev, 4ㅇ (ZMUM).

DISTRIBUTION. P. subventa is a common W Palaearctic species: Europe (to the east to Central European Russia and Russian Caucasus); N Africa: Morocco; Near East: Turkey. The easternmost known locality is Azerbaijan $\left(48.78^{\circ} \mathrm{E}\right)$. Introduced in the Nearctic region.

REMARKS. The variability in comparison with $P$. rufiventris is given below. In vast majority of cases it is necessary to choose between $P$. rufiventris and $P$. subventa - two the most common European species. At the same time these two 
species are the most variable in the $P$. pallida group $(P$. pallida and $P$. kowarzii are variable only in colour pattern of the thorax, other species are known by very limited series).

\section{$P$. rufiventris}

Aristal hairs. $O^{7}+$ : $0.75-1.3 \mathrm{x}$ as long as width of postpedicel.

Pedicel. $\sigma^{7}+$ : yellow or dark.

Interfrontal setae. + : absent.

Inclinate frontal setae. $O^{7}: 5-6$ pairs of inclinate frontal setae (either strong and weak ones) confined to lower third of frons.

Thoracic colour pattern. $O^{7}$ : In dark specimens (about $65 \%$ ) the colour pattern is similar to yellowish males of $P$. subventa i.e. only postpronotal lobe yellow, scutellum usually darkened at base. In yellow specimens (about 35\%) the sides of the scutum outside the intraalar rows and pleura somewhere yellow, scutellum usually yellow. + : the colour pattern as in yellow males.

Presutural $a c$ setae. $O^{7}: 1$ pair, rarely 2 pairs $(6 \%)$..+1 pair or absent (30\% specimens).

Katepimeron. $0^{\text {? }}$ : with $1-2$ setulae, rarely bare in $2 \%$ of accurately mounted specimens; more frequent in the specimens in bad condition or collected by YPT or Malaise trap; $q$ with 1 hair or bare ( $40 \%$ specimens). The typical situation is that the setulae are present or absent on the katepimeron on both sides of the thorax; specimens with the setula presents on one side and absents on the other are rare. Such distribution indicates that the setulae on the katepimeron were not broken but were initially absent in almost half of females.

Meron. O'o : bare.

Crossveins on wing. $\sigma^{\top} O$ : indistinctly infuscated.

t1. $\sigma^{7}+$ : without $p$ seta.

t2. $0^{2}+$ : with 3 rarely $4(7 \%) p$ setae.

\section{Ecology}

No information was published on ecology of $P$. bitincta, $P$. luteovittata, $P$. regalis or $P$. rubriventris, the authors of this paper also have never observed these species personally.

$P$. subventa is a typical visitor of excrements or carrion. Other 3 species: $P$. kowarzii, $P$. pallida and $P$. rufiventris are not specially attracted to rotten organic, although can visit it occasionally. Their typical habitat is grass or low brushes in forest. $P$. pallida distinctly prefers grass, while $P$. kowarzii prefers leaves of bushes at a height of 1-2 m, P. rufiventris may be found either on grass or bushes.

Identification key for Palaearctic Phaonia PaLlida GROUP

1. $d c 2+3$. (Strong prst ac always absent in both sexes. Meron and katepimeron always bare) .......................... 2

- $d c 2+4$...

2. Femora and tibiae entirely yellow. Palpi yellow. Calyptrae yellow. At least postpronotal lobe translucent yellow $\left(\sigma^{\top}\right.$, Fig. 11), in $q$ thorax usually broadly yellow as on Fig. 12. $\bigcirc^{7}$ : Postpedicel remarkably yellowish-white (Fig. 11) kowarzii Shnabl

- Fore femur and tibia mostly dark; mid and hind legs with femora darkened at apex and tibiae darkened at base. Palpi dark. Calyptrae brown. Thorax entirely dark in both

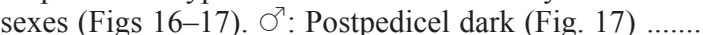

rubriventris Emden

3. Radial node on lower side of wing with 2-4 setulae. Thorax normally entirely yellow with whitish dusting on
Thus, we offer the direct comparison of $P$. rufiventris and $P$. subventa together with the data on variability from hundreds of examined specimens.

\section{P. subventa}

Aristal hairs. $\sigma^{7}+$ : $0.5-0.75 \mathrm{x}$ as long as width of postpedicel.

Pedicel. $\sigma^{7}$ : dark. + : dark, rarely (4\%) yellow(ish).

Interfrontal setae. + : present, sometimes $(17 \%)$ absent.

Inclinate frontal setae. $0^{7}: 7-10$ pairs of inclinate frontal setae (either strong and weak ones) extend to middle of frons.

Thoracic colour pattern. $\sigma^{7}$ : Thorax entirely dark, including postpronotal lobe and dorsal surface of the scutellum, only sides of scutellum yellow $(30 \%)$; or thorax dark, but postpronotal lobe yellow(ish) and dorsal surface of scutellum mostly yellow, only its base darkened $(70 \%)$. + : Thorax entirely dark, including postpronotal lobe and dorsal surface of the scutellum (14\%); or thorax dark, but postpronotal lobe yellow(ish) (86\%), in this form dorsal surface of scutellum mostly yellow, only its base darkened, rarely (2\%) scutellum entirely yellow.

Presutural $a c$ setae. $0^{7}+: 2$ or $3(14 \%)$ pairs.

Katepimeron. $\sigma^{70}+$ : bare.

Meron. $0^{7}$ : with 1-5 setulae along lower border of posterior spiracle (absent/broken in 3\%). + : with 1-3 hairs or bare in $27 \%$

Crossveins on wing. $\sigma^{7}+$ : distinctly infuscated.

t1. $\sigma^{7}$ : without $p$ seta (or with $1 p: 19 \%$ ); : with 1(2) $p$, rarely without $p$ setae $(5 \%)$.

t2. $0^{7}+$ : with 2 or $3(10 \%) p$ setae.

scutum. (Katepimeron setulose as on Fig. 1b, meron bare. $t 1$ without seta. Aristal hairs longer than width of postpedicel).

- Radial node on lower side of wing bare. Thorax, especially scutum more or less extensively darkened ............. 5

4. Notopleuron densely haired. Strong prst ac absent. Hairs on occiput and pleura partly yellow (katepimeron with all hairs yellow). Large species, body length 9-11 mm .....

regalis Stein

- Notopleuron bare. Two pairs of strong prst ac. All hairs on occiput and pleura black. Medium size species, body length $6-8 \mathrm{~mm}$................................... pallida Fabricius

5. Katepimeron usually with 1-2 minute hairs (Fig. 1b), meron always bare. Crossveins not infuscated. $0^{7}: 4-6$ pairs of inclinate frontal setae (either strong and weak ones) confined to lower third of frons. prst ac - 1(2) pairs. + : Frons without interfrontals. $t 1$ without $p$. prst ac

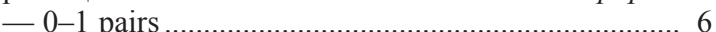

- Katepimeron always bare, meron usually with 2-6 minute hairs along lower border of posterior spiracle (Fig. 1a). Crossveins distinctly infuscated. $0^{7}$ : 7-10 pairs of inclinate frontal setae (either strong and weak ones) extend over the middle of frons. prst ac $-2-3$ pairs. O : Frons usually with interfrontals. $t 1$ usually with $p$. prst $a c-2$ pairs ......

6. $f 1$ darkened. Tergites 5 entirely and tergite 4 partly glossy black, tergite 3 often darkened on posterior border. Disc of scutum with only thin grey dusting. Scutellum always pure amber-yellow. $\sigma^{7}$ : Cercal plate with strong sclerotization and halves of cerci narrowed at apex (Fig. 3). . : Strong prst $a c$ absent luteovittata Shinonaga et Kano 
- $f 1$ yellow. Tergites yellow, at most slightly darkened somewhere. Disc with distinct grey dusting. Scutellum yellow or darkened at base. $\sigma^{7}$ : Cercal plate with weak sclerotization, and halves of cerci bidental at apex (Fig. 4). + : Strong prst ac usually present rufiventris Scopoli

7. Aristal hairs $1.5 \mathrm{x}$ as long as width of postpedicel. Back of the head (lower occiput and postgenae) covered with golden setulae. Scutum densely grey dusted, in posterior view on presutural part of scutum, the width of pair of black submedian vittae (together) $0.5 \mathrm{x}$ as wide as width of median grey vitta between them (Fig. 8). Abdomen evenly (though thinly) whitish dusted with distinct undusted median vitta. $t 2$ with 3(4) $p$ setae. $\sigma^{\prime}$ : Cheeks wide, 1.5$2 \mathrm{x}$ wider than width of $f 1 . f 2$ with spine-like ventral setae (Fig. 9). Pedicel yellowish. : Fronto-orbital plates and parafacias densely and evenly whitish dusted; frons thinly whitish dusted (Fig. 10)

bitincta Rondani

- Aristal hairs at most $0.75 \mathrm{x}$ as long as width of postpedicel. All occipital setulae black. Scutum thinly dusted, partly shining black, in posterior view on presutural part of scutum, the width of pair of black submedian vittae (together) wider than width of median grey vitta between them (Fig. 5). Abdomen mostly shining yellow (sometimes partly darkened), median vitta indistinct. $t 2$ with
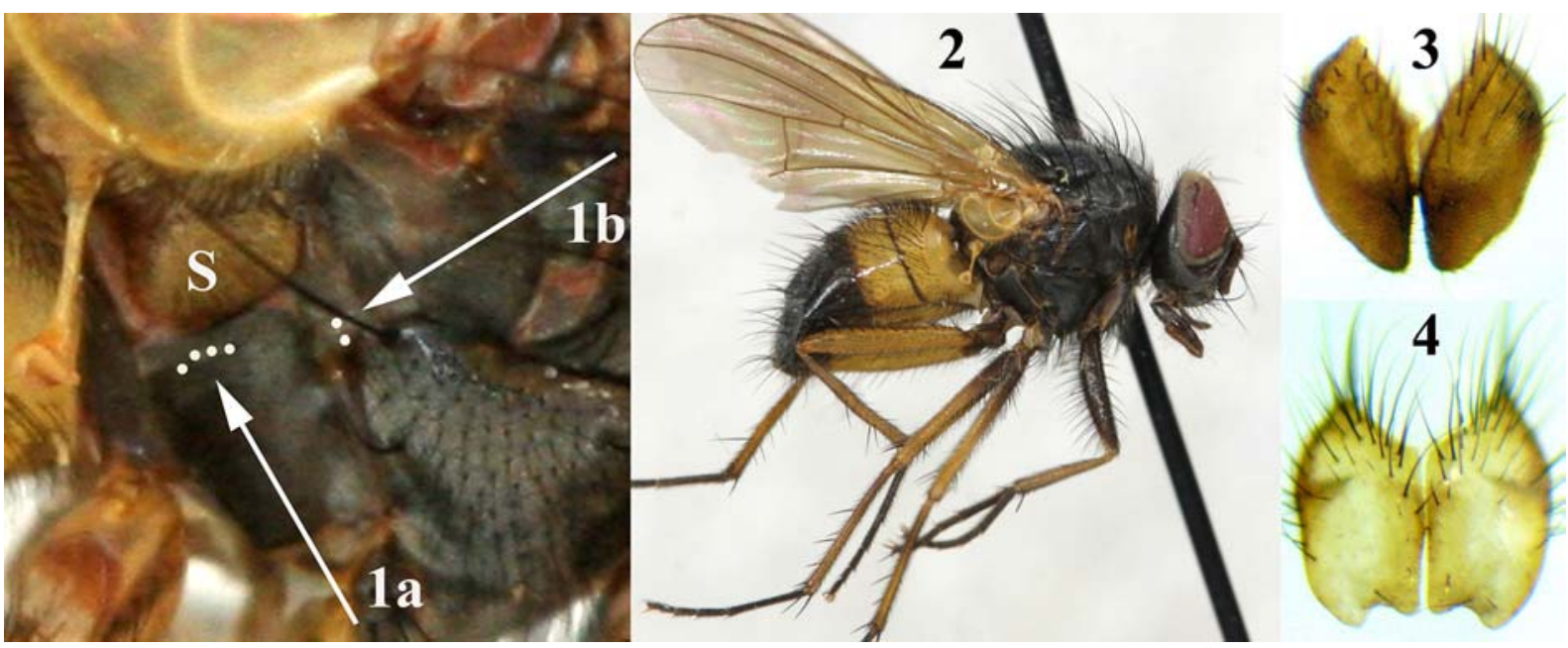

Figs 1-4. Phaonia spp: 1-3 - P luteovittata ; $4-P$ rufiventris; 1 - location of hypopleural hairs; 2 - habitus, male; $3-4-$ cercal plate; $1 \mathrm{a}$ - on meron along the lower border of posterior spiracle, $1 \mathrm{~b}$ - on katepimeron, $\mathrm{S}$ - posterior spiracle

Рис 1-4. Phaonia spp: 1-3-P. luteovittata; 4 - P. rufiventris; 1 - расположение гипоплевральных волосков; 2 - внешний вид, самец; 3-4 - церки; 1a — на мероне вдоль нижней границы заднего дыхальца; $1 \mathrm{~b}$ — на катэпимероне, S — заднее дыхальце.

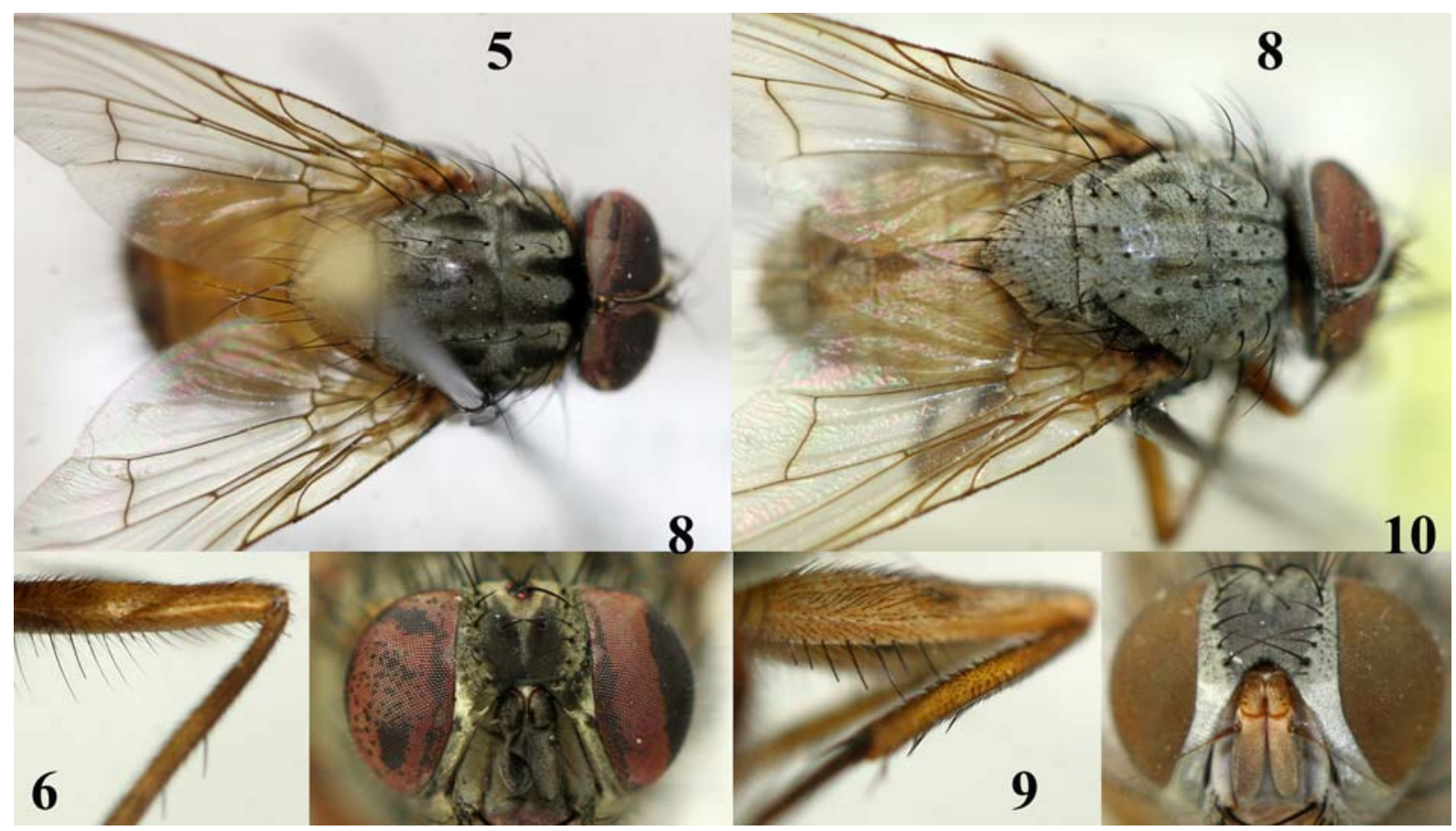
head.

Figs 4-10. Phaonia spp: 5-7 - P. subventa; 8-10-P. bitincta; 5, 8- male, dorsal view; 6, 9 - male $f 2$, anterior view; 7, 10 - female

Рис 4-10. Phaonia spp: 5-7 - P. subventa; 8-10-P. bitincta; 5, 8 - самец, сверху; 6, 9 - f2 самца, спереди; 7, 10 — голова самки. 

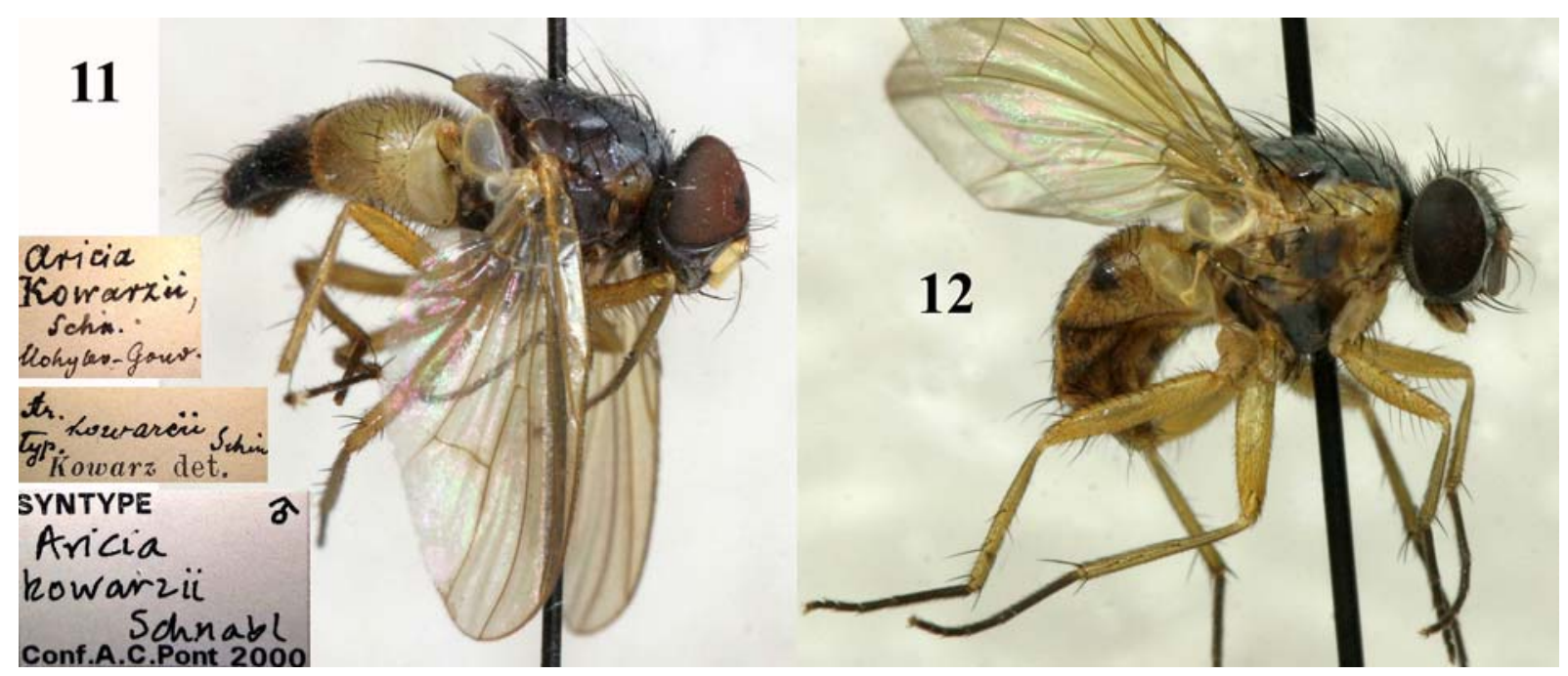

Figs 11-12. P. kowarzii: 11 - male syntype; 12 - female.

Рис 11-12. P. kowarzii: 11 - самец, синтип; 12 - самка.
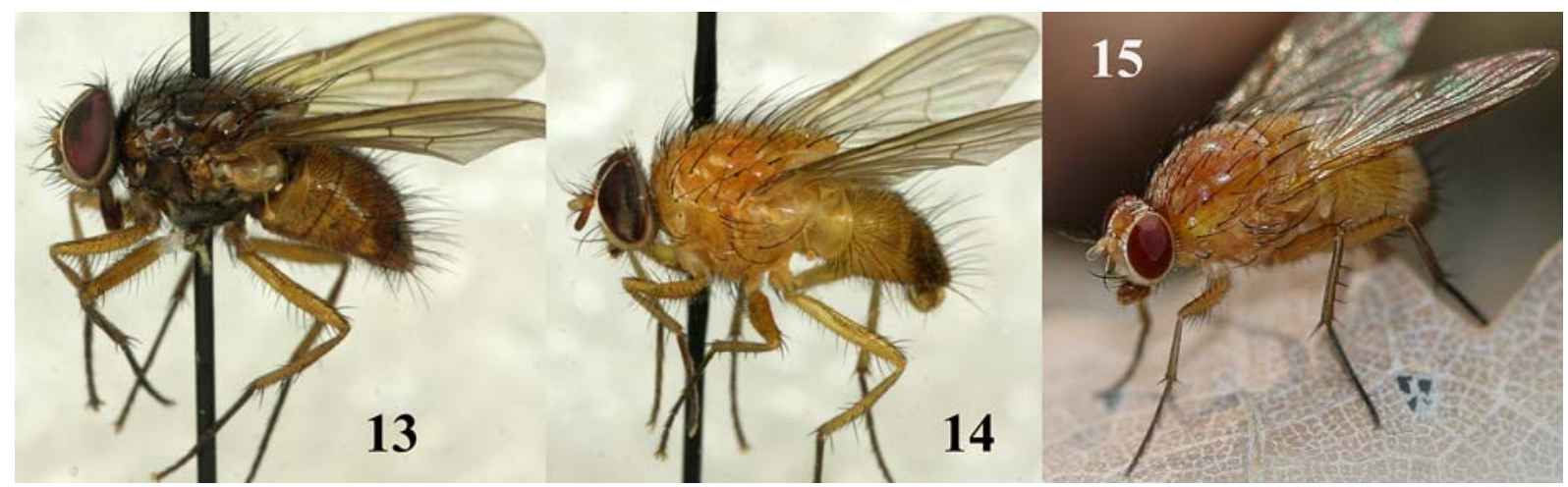

Figs 13-15. P. pallida: 13 - male, dark form; 14 - typical male; 15 - typical female.

Рис 13-15. P. pallida: 13 - самец, тёмная форма; 14 - самец типичной окраски; 15 - типичная самка.
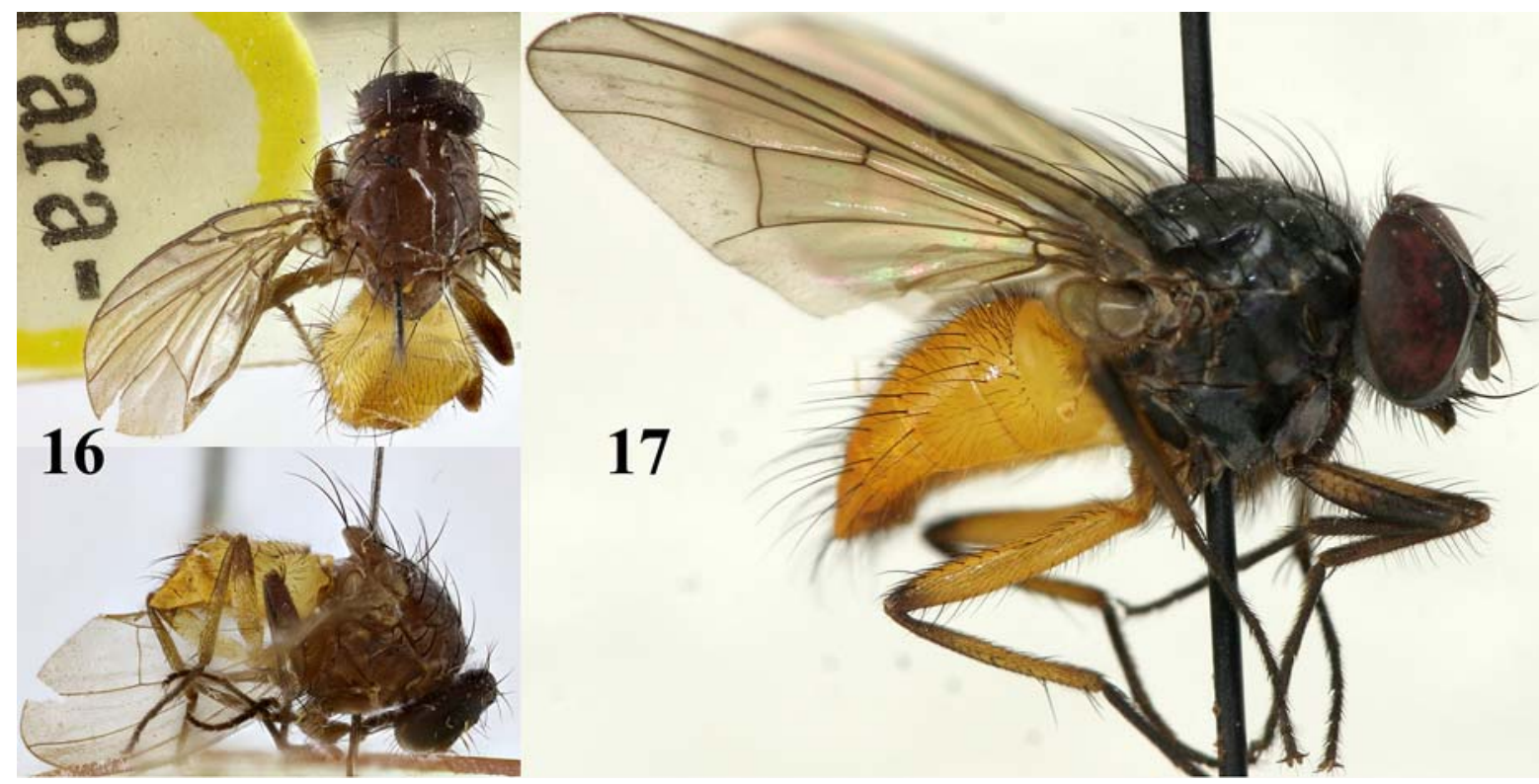

Figs 16-17. P. rubriventris: 16 - female paratype (photo: Nigel Wyatt); 17 - male.

Рис 16-17. P. rubriventris: 16 - самка, паратип (фото: Nigel Wyatt); 17 - самец. 
2(3) $p$ setae. $\sigma^{7}$ : Cheeks narrower, about as wide as width of $f 1$. $f 2$ with fine ventral setae (Fig. 6). Pedicel dark. + : Frons, fronto-orbital plates and parafacials dark (parafacials with whitish spot at level of pedicel (Fig. 7).

subventa Harris

ACKNOWLEDGEMENTS. We thank Olga Ovchinnikova and Galina Suleymanova (ZIN, St-Petersburg); Vera Sorokina (ISEA, Novosibirsk) for the loan of material. We are very grateful to Verner Michelsen (ZMUC, Copenhagen); Adrian Pont (OUMNH, Oxford); Nigel Wyatt (BMNH, London) for the examination of specimens in the collections in their care. We thank Oleg Kosterin (Novosibirsk) and Andrey Ozerov (Moscow) for their corrections and advices. Alexander Erofeev and Maria Yanbulat (Moscow) helped us a lot during the work on this paper. We are especially grateful to all collectors for the rich material listed above.

\section{References}

d'Assis-Fonseca ECM. 1968. Diptera Cyclorrhapha Calyptrata, Muscidae // Handbook for the Identification of British Insects. 10. 4(b). London: Royal Entomological Society. 118 pp.
Gregor F., Rozkošný R., Barták M., Vaňhara J. 2002. The Muscidae (Diptera) of Central Europe // Folia Facultatis Scientiarum Naturalium Universitatis Masarykianae Brunensis, Biologia. 107. $280 \mathrm{pp}$

Ma Z., Xue W., Feng Y. 2002. Fauna Sinica Insecta. Vol.26. Diptera: Muscidae II, Phaoniinae I. Beijing: Science Press. 421 pp.

Emden van F.I. 1965. The fauna of India and the adjacent countries. Diptera, Vol.7. Muscidae, Pt.1. Delhi: Government of India. 647 pp.

Hennig W. 1963. Muscidae. [Part, Lieferung 233, 234 and 241] // Lindner E. (Hrsg.). Die Fliegen der Palaearktischen Region. Stuttgart: Schweizerbart. Lfg.63b. S.772-899.

Pont A.C. 1986. Family Muscidae // Soós A., Papp L. (eds.). Catalogue of Palaearctic Diptera. Vol.11. Budapest: Akadémia Kiadó. P.57-215.

Shinonaga S. 2003. Monograph of the Muscidae of Japan. Tokyo: Tokai University Press. 347 pp.

Veselkin G.A. 1966. [Flies (Diptera), satellites of domestic animals and man, in the southern part of the Tyumen Region] // Entomologicheskoe Obozrenie. Vol. 45. No.4. P.779-792 [in Russian].

Zinovjev A. G. 1981. [On the classification of the Palearctic flies of the genus Phaonia R.-D. (Diptera, Muscidae)] // Entomologicheskoe Obozrenie. Vol.60. No.3. P.686-697 [in Russian]. 